\title{
Preventive Effect of Isometric Contraction Exercise on Disuse Muscle Atrophy Using Tail Suspension Mice
}

\author{
YUMI KAWAHARA ${ }^{1)}$, TAKESHI NiKAWA ${ }^{2)}$, KATSUYA HIRASAKA ${ }^{2)}$, TAKASHI MIYASHITA ${ }^{1)}$, \\ KATSUKO KATAOKA ${ }^{3)}$, LOUIS YUGE ${ }^{1)}$ \\ 1)Division of Bio-Environmental Adaptation Sciences, Graduate School of Health Sciences, \\ Hiroshima University: 1-2-3 Kasumi, Minami-ku, Hiroshima 734-8551, Japan. \\ TEL +81 82-257-5425, FAX +81 82-257-5344, \\ E-mail: ryuge@hiroshima-u.ac.jp \\ 2)Department of Nutrition, The University of Tokushima Faculty of Medicine \\ ${ }^{3)}$ Department of Histology and Cell Biology, Graduate School of Biomedical Science, \\ Hiroshima University
}

J. Phys. Ther. Sci.

20: $39-44,2008$

\begin{abstract}
In the present study, we demonstrated the preventive effect of isometric contraction exercise on disuse muscle atrophy with tail suspension immobilization model mice. Mice were divided into three groups: tail suspension alone (group TS), addition of exercise to tail suspension (group EX), and control (group C). Muscle mass (muscle weight divided by body weight) of soleus (SOL) and gastrocnemius (GAS) muscles were lower in group TS than in group C. In group EX, muscle mass of SOL increased compared to group TS at 2 weeks. Histological examination indicated that group TS mice developed muscle atrophy in both SOL and GAS, while group EX mice did not develop atrophy in SOL. Moreover, this study first demonstrated that muscle mass recovered from atrophy in group EX at 2 weeks. Myosin heavy chain expression was significantly higher in group EX than in group TS at 2 weeks. Our results suggest that isometric contraction exercise prevented disuse muscle atrophy in the soleus muscle.
\end{abstract}

Key words: Disuse muscle atrophy, Isometric contraction exercise, Tail suspension

(This article was submitted Aug. 22, 2007, and was accepted Oct. 19, 2007)

\section{INTRODUCTION}

It is well known that muscle becomes hypertrophic by exercise, and atrophic by decrease of muscle activity for a long time. Hypertrophy is a reaction that takes full advantage of muscle strength in overload. On the other hand, atrophy is a reaction to the regulation of resting energy expenditure to cut down on waste, because muscle consumes a lot of energy, even in the resting state. Muscle atrophy is caused by decreased muscle activity. Various physical stimulation methods, such as stretching, electric stimuli, heating and cooling, have been applied in a clinical situation as well as in a spaceflight for preservation of muscle strength and preventing disuse muscle atrophy.

Isometric contraction exercise is also used widely to promote muscle endurance to prevent disuse muscle atrophy. The advantage of the isometric method is that it can be used for patients on bed rest and/or in plaster casts. Previous reports on the effect of isometric contraction exercise have been limited to normal rats or mice with experimentally induced muscle atrophy ${ }^{3,4)}$. Tasaki et al. ${ }^{4)}$ reported that muscle atrophy was partially inhibited in tail suspension mice with isometric contraction. 
However, they reported soleus muscles (SOL) and extensor digitorum longus (EDL), but not gastrocnemius muscle (GAS), and the isometric exercise was performed after muscle atrophy had been established. Other reports have also demonstrated the effectiveness of isometric contraction exercise for muscle atrophy from the standpoint of muscle mass, fiber diameter and strength $^{5-7)}$. However, there are few reports in which isometric exercise was examined during the progression of muscle atrophy using molecular biological analysis.

We studied the effect of isometric contraction exercise simultaneously with the disuse model of immobilization, and examined histological and molecular biological methods to investigate whether isometric exercise can prevent disuse muscle atrophy.

\section{METHODS}

Forty-eight male C57BL/6 mice (eight weeks old, average weight $22.6 \mathrm{~g}$ ) were used. The animals were housed under standard laboratory conditions at a temperature of $23 \pm 1^{\circ} \mathrm{C}$, relative humidity of $55 \pm$ $5 \%$, and a 12-hour light/dark cycle. Mice were provided with food and water ad libitum. The experimental protocol of this study was approved by the Institute of Laboratory of Animal Science of Hiroshima University.

Mice were randomly divided into three groups (sixteen mice per group): tail suspension alone (group TS), addition of exercise to tail suspension (group EX), and control (group C). In group TS and EX, the tails of the mice were suspended from the ceiling with medical tape to keep their hind limbs off the ground (Fig. 1A). Mice could move in the laboratory cage using their forelimbs to get food and water. In group EX, mice were loaded with $75 \%$ of body weight load at their tail and were allowed to cling to a wire mesh slope at 80 degrees as described by Tasaki et al. ${ }^{4)}$ (Fig. 1B). This exercise was performed for 20 minutes a day, five times a week. Control mice were raised normally, with no tail suspension and no enforced exercise.

At 1 or 2 weeks (wk), eight mice of each group were sacrificed by cervical dislocation and their body weights were measured. GAS, SOL, and EDL were excised from both legs and weighed for wetweight.

Each left side muscle of the medial head was
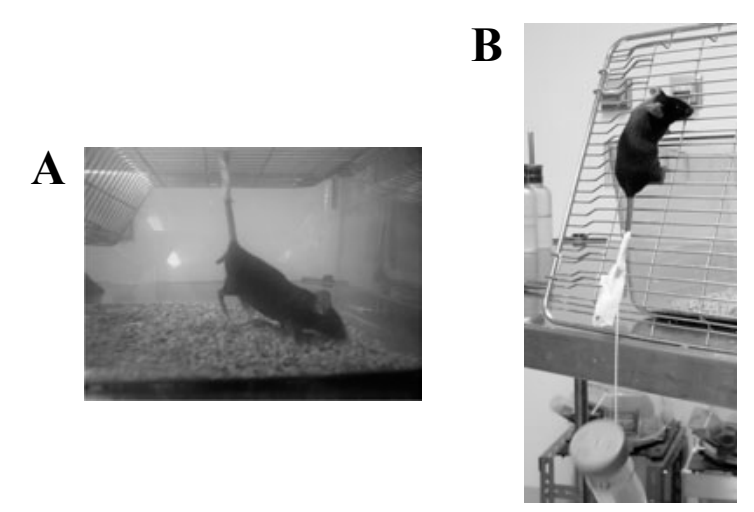

Fig. 1. Pictures of a tail suspended (A) and exercising (B) mouse.

fixed in $10 \%$ formalin for one day, then dehydrated and embedded in paraffin. Five $\mu \mathrm{m}$ thick sections were stained with hematoxylin and eosin (H\&E). Twenty-four microscopic photographs (three pictures per mouse, eight mice per group) were randomly taken at $\times 40$ in magnification and eight pictures were randomly selected. The crosssectional area (CSA) of each myofiber was measured in 80 myofibers selected at random and totaled for each pictures (number of measured myofibers in each muscle of each mouse $=640$ ) using NIH image 1.62 (National Institutes of Health, Bethesda, Maryland, USA).

The right side muscles were kept frozen until analysis. Each muscle was homogenized in $20 \mathrm{mM}$ tris- $\mathrm{HCl}$ buffer, $\mathrm{pH} 7.5$, containing $150 \mathrm{mM} \mathrm{NaCl}$, $1 \%$ triton-X100, complete protease inhibitor cocktail tablet (Roche Diagnostics, Basel, Switzerland), and $10 \mu \mathrm{M}$ epoxomicin. After centrifugation at $12,000 \mathrm{rpm}$ for $15 \mathrm{~min}$ at $4{ }^{\circ} \mathrm{C}$, proteins in the supernatant (soluble fraction) were subjected to western blot analysis. Protein content was quantified using the Bio-Rad protein assay (Bio-Rad, Hercules, California, USA) with albumin as the standard. Proteins ( $40 \mu \mathrm{g} /$ lane) were separated by 6\%-polyacrylamide gel electrophoresis and transferred to a nitrocellulose membrane (Hybond ${ }^{\mathrm{TM}}$-ECL; Amersham Biosciences UK Ltd., Buckinghamshire, England). The membrane was blocked overnight at $4^{\circ} \mathrm{C}$ with tris-buffered saline containing $0.05 \%$ Tween 20 and $5 \%$ skim milk. Blots were incubated with primary antibody at 1:500 dilution of anti-skeletal MHC (Sigma, Saint Louis, Missouri, USA). After application of the secondary antibody, detection was carried out using an enhanced 
Table 1. Effects of isometric contraction exercise on muscle wet-weight divided by body weight

\begin{tabular}{|c|c|c|c|c|c|c|c|}
\hline & \multicolumn{3}{|c|}{$1 \mathrm{wk}$} & \multicolumn{3}{|c|}{$2 \mathrm{wk}$} \\
\hline & & $\begin{array}{l}\text { group C } \\
(n=8)\end{array}$ & $\begin{array}{l}\text { group TS } \\
(\mathrm{n}=8)\end{array}$ & $\begin{array}{l}\text { group EX } \\
(\mathrm{n}=8)\end{array}$ & $\begin{array}{l}\text { group C } \\
(n=8)\end{array}$ & $\begin{array}{l}\text { group TS } \\
(\mathrm{n}=8)\end{array}$ & $\begin{array}{l}\text { group EX } \\
(\mathrm{n}=8)\end{array}$ \\
\hline \multicolumn{2}{|c|}{ Body weight (BW; g) } & $21.5 \pm 0.8$ & $17.1 \pm 2.7^{*}$ & $19.6 \pm 1.6^{\dagger}$ & $23.5 \pm 0.9$ & $21.0 \pm 0.9^{*}$ & $18.0 \pm 2.2^{*, \dagger}$ \\
\hline GAS & $\begin{array}{l}\text { Wet weight (WW; mg) } \\
\text { WW/BW }\end{array}$ & $\begin{aligned} 108.5 & \pm 12.9 \\
5.04 & \pm 0.53\end{aligned}$ & $\begin{array}{l}73.3 \pm 17.1^{*} \\
4.29 \pm 0.33^{*}\end{array}$ & $\begin{array}{l}86.3 \pm 19.6^{\dagger} \\
4.42 \pm 0.29^{*}\end{array}$ & $\begin{aligned} 112.2 & \pm 11.4 \\
4.77 & \pm 0.39\end{aligned}$ & $\begin{array}{l}79.1 \pm 6.1^{*} \\
3.77 \pm 0.31^{*}\end{array}$ & $\begin{array}{l}72.8 \pm 18.0^{*} \\
4.06 \pm 0.43^{*}\end{array}$ \\
\hline SOL & $\begin{array}{l}\text { Wet weight (WW; mg) } \\
\text { WW/BW }\end{array}$ & $\begin{aligned} 7.6 & \pm 1.1 \\
0.33 & \pm 0.05\end{aligned}$ & $\begin{array}{c}3.8 \pm 1.0 * \\
0.22 \pm 0.06^{*}\end{array}$ & $\begin{aligned} 5.1 & \pm 0.8^{*, \dagger} \\
0.26 & \pm 0.04^{*}\end{aligned}$ & $\begin{aligned} 7.8 & \pm 0.8 \\
0.33 & \pm 0.03\end{aligned}$ & $\begin{array}{c}3.6 \pm 0.6^{*} \\
0.17 \pm 0.03^{*}\end{array}$ & $\begin{aligned} 4.4 & \pm 0.6^{*} \\
0.25 & \pm 0.04^{*, \dagger}\end{aligned}$ \\
\hline EDL & $\begin{array}{l}\text { Wet weight (WW; mg) } \\
\text { WW/BW }\end{array}$ & $\begin{aligned} 8.0 & \pm 1.0 \\
0.40 & \pm 0.09\end{aligned}$ & $\begin{aligned} 6.1 & \pm 1.1^{*} \\
0.36 & \pm 0.05\end{aligned}$ & $\begin{aligned} 7.0 & \pm 0.8^{*, \dagger} \\
0.36 & \pm 0.03\end{aligned}$ & $\begin{aligned} 8.9 & \pm 0.8 \\
0.38 & \pm 0.03\end{aligned}$ & $\begin{aligned} 7.4 & \pm 0.5^{*} \\
0.35 & \pm 0.03\end{aligned}$ & $\begin{aligned} 6.5 & \pm 1.8^{*} \\
0.36 & \pm 0.06\end{aligned}$ \\
\hline
\end{tabular}

All values are given as mean $\pm \mathrm{SD}$. * vs. group $\mathrm{C}(\mathrm{p}<0.01)$. † vs. group TS $(\mathrm{p}<0.01)$.

chemiluminescence system (Amersham, Little Chalfont, England).

All values are given as the mean $\pm \mathrm{SD}$ of the eight individual samples per group and were analyzed by two-way ANOVA (StatView Ver. 5.0, SAS Institute Inc., Cary, North Carolina, USA). Tukey's test was used to determine differences among the groups. A P value of $<0.01$ was considered to be statistically significant.

\section{RESULTS}

Body weights were lower in groups TS and EX than in group $\mathrm{C}$ (Table $1, \mathrm{n}=8$ per each groups). For evaluating muscle mass, the wet-weight of each muscle divided by body weight $(\mathrm{mg} / \mathrm{g})$ significantly decreased with time in groups TS and EX as compared to group C (Table 1) in both GAS and SOL. However, in group EX, the reduction was slighter than in group TS, especially in SOL. EDL showed no change among the groups, and was excluded from further examinations.

GAS and SOL muscles were examined histologically by $\mathrm{H} \& \mathrm{E}$ staining. In group $\mathrm{C}$, muscle fibers were polygonal and approximately uniform in size, and had their nuclei at the periphery in both GAS and SOL (Fig. 2A-B, Fig. 3A-B). No changes were observed between $1 \mathrm{wk}$ and $2 \mathrm{wk}$ in group $\mathrm{C}$; muscle atrophy and regeneration were not found. In group TS, the number of polygonal muscle fibers decreased, and their size was variable in GAS (Fig. 2C-D) and SOL (Fig. 3C-D). Moreover, the size of the muscle fibers decreased with time. Thinner muscle fibers were evident among muscle fibers of normal thickness at $2 \mathrm{wk}$. However, in group EX, muscle atrophy was less prominent in both GAS and SOL (Fig. 2E-F, Fig. 3E-F). These observations were also confirmed by CSA of GAS and SOL (Table 2, $\mathrm{n}=640$ per group). Muscle atrophy developed in groups TS and EX at $1 \mathrm{wk}$. At $2 \mathrm{wk}$, in both GAS and SOL atrophy progressed in group TS, whereas in group EX, both muscles recovered from atrophy.

In SOL, MHC protein expression decreased in group TS and EX compared to group $\mathrm{C}$ at $1 \mathrm{wk}$ (Fig. $4 \mathrm{~B}$, Table 2). In SOL of group EX, there was significantly higher expression than in group TS at 2 wk. No significant changes were found in the expression of MHC protein in GAS (Fig. 4A, Table 2).

\section{DISCUSSION}

Cellular differentiation can be switched on by both chemical and physical stimuli. Hormones and growth factors bind to specific receptors on the cell surface responsible for inducing cell proliferation and differentiation ${ }^{8)}$. Physical stimuli, such as a mechanical stress and electrical stimulation, are particularly important in the differentiation and maintenance of contractile cells $\mathrm{s}^{1,2,8,9)}$. We previously reported that the differentiation of myoblasts containing intracellular magnetic microparticles occurred earlier when they were cultured in a magnetic field while being stretched ${ }^{1)}$. This in vitro experiment demonstrated that mechanical stretch induced muscle cell differentiation, and electrical stimulation also induced myoblast differentiation ${ }^{9}$. In the present study, we investigated the effect of isometric contraction in vivo using disuse muscle atrophy model mice. 

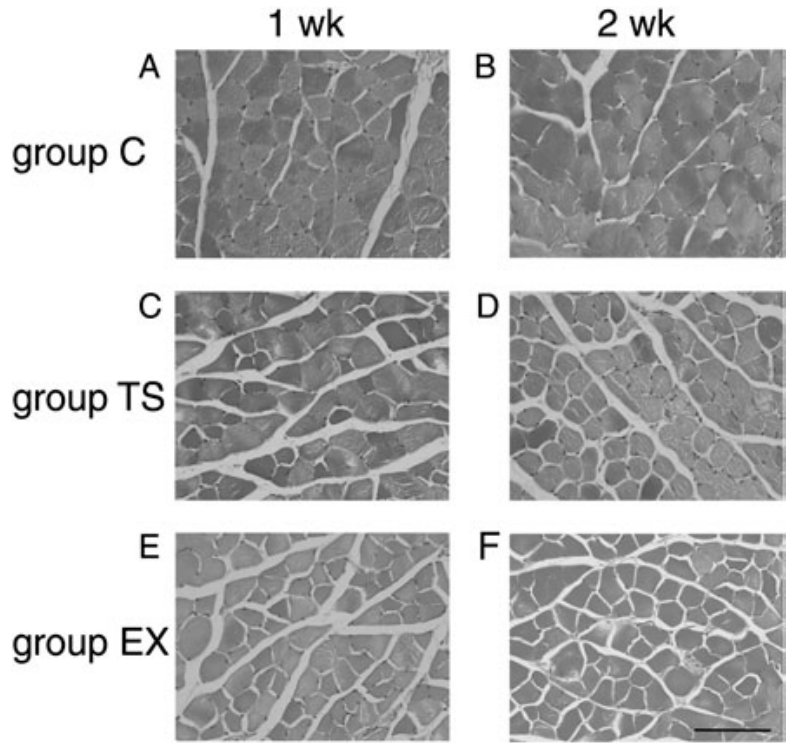

Fig. 2. Histological images of GAS. The diameter of muscle fibers was uniform in group C (A, B). In group TS, smaller muscle fibers increased with time (C, D). Group EX showed a similar tendency but the change was smaller (E, F). Scale bar, $100 \mu \mathrm{m}$. CSA of muscle fibers is shown in Table 2.
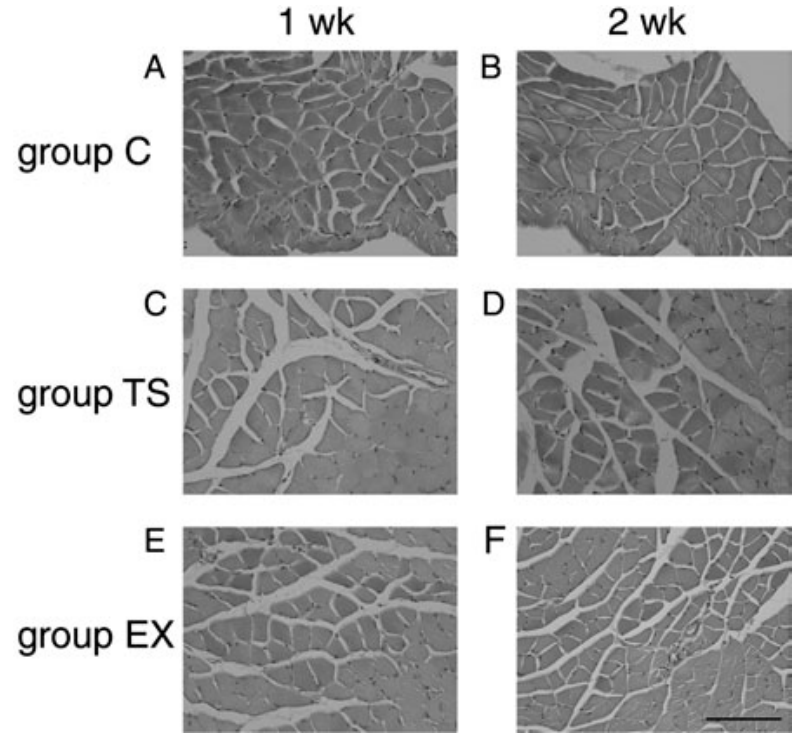

Fig. 3. Histological images of SOL. The diameter of muscle fibers was uniform in group $\mathrm{C}(\mathrm{A}, \mathrm{B})$. In group TS, smaller muscle fibers increased with time (C, D). Group EX showed a similar tendency but the change was smaller (E, F). Scale bar, $100 \mu \mathrm{m}$. CSA of muscle fibers is shown in Table 2.

Table 2. Effects of isometric contraction exercise on CSA of muscle fiber and density rate of MHC

\begin{tabular}{|c|c|c|c|c|c|c|}
\hline & \multicolumn{3}{|c|}{$1 \mathrm{wk}$} & \multicolumn{3}{|c|}{$2 \mathrm{wk}$} \\
\hline & group $\mathrm{C}$ & group TS & group EX & group $\mathrm{C}$ & group TS & group EX \\
\hline \multicolumn{7}{|c|}{ CSA of muscle fiber $\left(\mu \mathrm{m}^{2}\right)(\mathrm{n}=640)$} \\
\hline GAS & $2809.8 \pm 614.2$ & $2190.6 \pm 758.4$ & $2165.8 \pm 657.4$ & $2775.2 \pm 351.5$ & $1797.2 \pm 555.6$ & $2553.9 \pm 632.6$ \\
\hline SOL & $1820.7 \pm 723.1$ & $1299.8 \pm 554.6$ & $1371.9 \pm 298.2$ & $1784.3 \pm 621.2$ & $1315.3 \pm 375.2 *$ & $1745.5 \pm 498.5^{\dagger}$ \\
\hline \multicolumn{7}{|c|}{ Density rate of $\mathrm{MHC}(\mathrm{n}=5)$} \\
\hline GAS & $1.00 \pm 0.04$ & $1.14 \pm 0.04$ & $1.17 \pm 0.01$ & $1.00 \pm 0.01$ & $1.03 \pm 0.01$ & $0.93 \pm 0.03$ \\
\hline SOL & $1.00 \pm 0.04$ & $0.70 \pm 0.03^{*}$ & $0.76 \pm 0.08^{*}$ & $1.00 \pm 0.04$ & $0.27 \pm 0.02 *$ & $0.73 \pm 0.03 *, \dagger$ \\
\hline
\end{tabular}

All values are given as mean \pm SD. *vs. group $C(p<0.01)$. † vs. group TS $(p<0.01)$.

We used a way to make mice cling to a wire mesh slope as an isometric contraction exercise as described by Tasaki et al. ${ }^{4)}$. The mice cling to the wire mesh slope, in order not to fall off, without joint movement. Tail suspension is commonly used for the disuse muscle atrophy model of immobilization or space-flight, and leads to a decrease in muscle weight. Muscle mass, in GAS and SOL, significantly decreased in groups TS and EX compared to group C (Table 1). But, that of EDL did not change in any group. Riley et al. ${ }^{12}$ ) suggested that EDL was kept in extension during tail suspension and therefore did not experience atrophy. Thus, we decided to exclude EDL from histological and molecular biological analysis.

In groups TS and EX, infiltration and abnormal accumulation of inflammatory cells, such as neutrophilic leukocytes and apoptotic bodies, were not observed. Thus, inflammation or destruction of muscle fibers was not found, which suggested that the exercise volume was suitable. The histological investigation also indicated that both TS and EX mice developed muscle atrophy, but that it was more intense in the former and the tendency was more evident in SOL than in GAS. The difference in atrophy between GAS and SOL might be related 

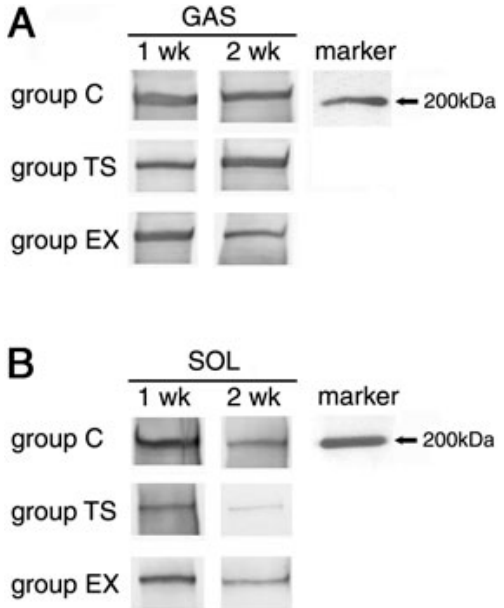

Fig. 4. Expression of MHC protein in GAS (A) and SOL (B). Each band density was measured by NIH image 1.62. MHC protein was low in group TS, especially in SOL. In group EX, MHC protein loss was suppressed. The density rate, ratios of groups TS and EX to group $\mathrm{C}$ is shown in Table 2.

to the fact that GAS is a fast-slow muscle, but SOL is a slow muscle. Isometric contraction effects muscle endurance improvement. Thus, the slow soleus muscle would be best protected by maintained isometric contractions, because the primary role of the soleus is to maintain posture against gravity by tonically contracting isometrically.

MHC is one of the main muscle proteins. Loss of MHC plays an important role in muscle atrophy from the aspect of muscle weight, contractile tension and contractile rate. In the present study, loss of MHC in SOL was suppressed in group EX at 2 wk compared to group TS (Fig. 4B), which suggests that isometric contraction suppressed the progression of disuse muscle atrophy. However the application of this effect seems limited, because the isometric contraction exercise used in this study was clinging to a wire mesh slope, an exercise which cannot be replicated by humans. Moreover, it is widely accepted that immobilization enables a transition from slower to faster MHC especially in slow muscles. We need to investigate which type of MHC was changed in the exercise model used in this study.

The final outcome of muscle protein during muscle atrophy depends on the balance between protein degradation and synthesis. Ikemoto et al. ${ }^{13}$ ) reported that tail suspension and space-flight lead to ubiquitination and degradation of MHC. DupontVersteegden et al. ${ }^{14)}$ recently reported that flywheel-based resistance exercise upregulated protein synthesis, but other atrophy-associated mechanisms, such as proteasomal subunit remodeling, elevation of apoptosis, and decrease in cellular proliferation, were unaffected. Other authors reported that tail suspension lead to increase oxidative stress resulting in muscle atrophy ${ }^{15-17)}$. Further studies are needed to elucidate the mechanisms of muscle atrophy and how isometric contraction exercise can prevent muscle atrophy.

In conclusion, we demonstrated that isometric exercise under tail suspension suppressed loss of muscle weight and protein to some extent compared to no exercise tail suspension. This is the first report to demonstrate the recovery of disuse muscle atrophy by exercise in an animal model of immobilization. Locomotor stimulation acts on the muscle through some signal pathways, and isometric contraction exercise suppressed the progression of disuse muscle atrophy and facilitated the recovery. A future study focusing on protein synthesis and degradation is required to provide scientific support for the physical treatment of disuse muscle atrophy.

\section{ACKNOWLEDGEMENTS}

We thank Reiko Yoshimoto, Akira Sasaki, ShuLiang $\mathrm{Wu}$, and Tomotaka Manabe, Division of BioEnvironmental Adaptation Sciences, Graduate School of Health Sciences, Hiroshima University, for technical support.

\section{REFERENCES}

1) Yuge L, Kataoka K: Differentiation of myoblasts is accelerated in culture in a magnetic field. In Vitro Cell Dev Biol, 2000, 36: 383-386.

2) Yuge L, Okubo A, Miyashita T, et al.: Physiol stress by magnetic force accelerates differentiation of human osteoblasts. Biochem Biophys Res Commun, 2003, 311: 32-38.

3) Exner GU, Staudte HW, Pette D: Isometric training of rats effects upon fast and slow muscle and modification by an anabolic hormone (nandrolone decanoate). Pflugers Arch, 1973, 345: 1-14.

4) Tasaki H, Okita M, Nakano J, et al.: Effects of isometric exercise after disuse muscle atrophy in mice. Rigaku ryohou tankyu, 1999, 2: 6-10 (in Japanese).

5) Horber FF, Hoopeler H, Scheidegger JR, et al.: Impact of physical training on the ultrastructure of midthigh 
muscle in normal subjects and in patients treated with glucocorticoids. J Clin Invest, 1987, 79: 1181-1190.

6) Akima $\mathrm{H}$, Kubo $\mathrm{K}$, Imai $\mathrm{M}$, et al.: Inactivity and muscle: effect of resistance training during bed rest on muscle size in the lower limb. Acta Physiol Scand, 2001, 172: 269-278.

7) Hurst JE, Fitts RH: Hindlimb unloading-induced muscle atrophy and loss of function: protective effect of isometric exercise. J Appl Physiol, 2003, 95: 14051417.

8) Alberts B, Johnson A, Lewis J, et al.: Molecular biology of the cell; 4th ed. New York: Garland Publishing, 2002, pp 831-852.

9) Kawahara Y, Yamaoka K, Iwata $M$, et al.: Novel Electrical Stimulation Sets the Cultured Myoblast Contractile Function to "ON". Pathobiology, 2007, 73: 288-294.

10) Yamanaka H, Kariya F, Tabata J, et al.: Morphological changes and myosin heavy-chain components of fast muscle in hidlimb unloaded adult rat. Journal of the Physiological Society of Japan, 2002, 64: 147-151 (in Japanese).

11) Saitoh A, Okumoto T, Nakano H, et al.: Age effect on expression of myosin heavy and light chain isoforms in suspended rat soleus muscle. J Appl Physiol, 1999,
86:1483-1489.

12) Riley DA, Slocum GR, Bain JL, et al.: Rat hindlimb unloading: soleus histochemistry, ultrastructure, and electromyography. J Appl Physiol, 1990, 69: 58-66.

13) Ikemoto $M$, Nikawa $T$, Takeda $S$, et al.: Space shuttle flight (STS-90) enhances degradation of rat myosin heavy chain in association with activation of ubiquitinproteasome pathway. FASEB J, 2001, 15: 1279-1281.

14) Dupont-Versteegden EE, Fluckey JD, Knox M, et al.: Effect of flywheel-based resistance exercise on processes contributing to muscle atrophy during unloading in adult rats. J Appl Physiol, 2006, 101: 202-212.

15) Lawler JM, Song W, Kwak HB: Differential response of heat shock proteins to hindlimb unloading and reloading in the soleus. Muscle Nerve, 2006, 33: 200207.

16) Haddad F, Adams GR, Bodell PW, et al.: Isometric resistance exercise fails to counteract skeletal muscle atrophy processes during the initial stages of unloading. J Appl Physiol, 2006, 100: 433-441.

17) Lawler JM, Song W, Demaree SR: Hindlimb unloading increases oxidative stress and disrupts antioxidant capacity in skeletal muscle. Free Radic Biol Med, 2003, 35: 9-16. 